\title{
BMJ Open Psychological and behavioural patterns of stigma among patients with type 2 diabetes: a cross-sectional study
}

\author{
Asuka Kato, ${ }^{1,2}$ Yuko Fujimaki, ${ }^{3}$ Shin Fujimori, ${ }^{3}$ Akihiro Isogawa, ${ }^{4}$ Yukiko Onishi, ${ }^{5}$ \\ Ryo Suzuki, ${ }^{6}$ Toshimasa Yamauchi, ${ }^{6}$ Kohjiro Ueki, ${ }^{6}$ Takashi Kadowaki, ${ }^{6}$ \\ Hideki Hashimoto $^{1}$
}

To cite: Kato A, Fujimaki Y, Fujimori S, et al.

Psychological and behavioural patterns of stigma among patients with type 2 diabetes: a crosssectional study. BMJ Open 2017;7:e013425.

doi:10.1136/bmjopen-2016013425

- Prepublication history for this paper is available online. To view these files please visit the journal online (http://dx.doi.org/10.1136/ bmjopen-2016-013425).

Received 11 July 2016 Revised 31 January 2017 Accepted 8 March 2017

CrossMark

For numbered affiliations see end of article.

Correspondence to

Dr Asuka Kato;

asukakato-tky@umin.ac.jp

\section{ABSTRACT}

Objectives: The aim of this study was to test the psychological and behavioural patterns of stigma (selfesteem and social participation) and their relationship to self-stigma, patient activation for engaging in selfcare and glycaemic control among patients with type 2 diabetes mellitus (T2DM).

Design: A cross-sectional study.

Setting: 2 tertiary-level hospitals and 2 secondarylevel hospitals in Japan.

Participants: A consecutive sample of 209 outpatients with T2DM. Inclusion criteria were as follows: presence of T2DM, age 20-74 years, no diagnosis of dementia and/or psychosis, and no need for urgent medical procedures.

Outcome measures: Study measures included a self-administered questionnaire to assess the Rosenberg Self-Esteem Scale (SES), the 3 subscales of 36-question Short Form Health Survey (SF-36; Social Function, Role Physical, Role Emotional), Self-Stigma Scale and Patient Activation Measure (PAM-13). Glycated haemoglobin was obtained from same day blood work. In our previous qualitative study, we found that psychological and behavioural patterns of stigma varied according to patients' levels of illness-related self-esteem as well as attitudes towards social participation. For quantitative consistency, we used the SES scale to measure self-esteem and the SF-36 subscales to measure social participation. We then divided participants into 4 groups by exhibited psychological and behavioural patterns: group A (high SES/high SF-36), group B (high SES/low SF-36), group $C$ (low SES/high SF-36) and group D (Iow SES/low SF36).

Results: Using analysis of covariance after controlling for age and sex, there was a significant difference in self-stigma levels between the four groups $(F[3,203]$ $=15.70, p<0.001)$. We observed the highest mean selfstigma levels in group D. Group D also had significantly lower PAM-13 scores than those of groups $A(p<0.001)$ and $B(p=0.02)$.

Conclusions: The psychological and behavioural pattern of group $D$ was found to be associated with higher levels of self-stigma and poorer patient activation for self-care.

\section{Strengths and limitations of this study}

- This is the first study to test the psychological and behavioural patterns of stigma (self-esteem and social participation) and their relationship to self-stigma, patient activation for engaging in self-care and glycaemic control among patients with T2DM.

- This study quantitatively demonstrated that there were differences between the distinct psychological and behavioural patterns of stigma by the two dimensions (self-esteem and social participation), which were derived from our previous qualitative study, suggesting that the group with low self-esteem/low social participation is associated with higher levels of self-stigma and poorer patient activation for self-care.

- Owing to the cross-sectional design of this study, causal relationships between self-stigma, self-esteem and attitudes towards social participation cannot be determined.

\section{INTRODUCTION}

Many people with type 2 diabetes mellitus (T2DM) experience diabetes-related stigma due to the fact that the condition is often perceived as a lifestyle-related disease by the general population. ${ }^{1-10}$ As a result, people with T2DM may feel ashamed of their illness and encounter discrimination, limited opportunities and negative stereotyping against them. ${ }^{6}$ Public stigma and self-stigma are two distinct but interrelated constructs. Public stigma refers to negative reactions of the general public towards a group based on stereotypical attributes distinguishing that group. ${ }^{11}$ Self-stigma, in contrast, refers to the internalisation of society's negative perceptions towards an illness by someone who has that particular illness. ${ }^{11} 12$ In order to internalise public stigma, an individual must first be aware that a public stigma for their illness exists, accept the stereotypes associated 
with their illness, and then develop a negative attitude towards the illness and thus themselves. ${ }^{11} 12$ This means that merely perceiving public stigma does not necessarily lead to the development of self-stigma. ${ }^{11} 12$

In this study, we decided to use the Self-Stigma Scale that consists of cognitive, affective and behavioural subscales. ${ }^{13}$ Since the Self-Stigma Scale was specifically designed to evaluate the self-stigma of groups that are not immediately identifiable, ${ }^{14}$ it is viewed as an appropriate tool for assessing the particularly concealable condition of T2DM. Using this scale, it was demonstrated that self-stigma is a significant predictor of decreased activation levels for engaging in self-care among patients with T2DM. ${ }^{15}$

It is a theoretically and empirically well-known fact that the two dimensions self-esteem and social participation are related to the ability to adjust to chronic illnesses in general. ${ }^{16}{ }^{17}$ Previous studies show that self-stigma is associated with self-esteem, which is defined as the degree to which an individual has a favourable or unfavourable opinion of himself/herself and finds himself/herself worthy or unworthy as a whole including their condition. ${ }^{13}{ }^{18-20}$ Depending on the degree of social participation, adjustment styles to illness differ. ${ }^{16}{ }^{17}$ For T2DM specifically, Kato $e t a l^{21}$ have found that patients' responses to self-stigma could be associated with social participation, which was defined as the degree to which patients are willing to participate in social life regardless of the severity of their condition.

In our previous qualitative study, ${ }^{21}$ we showed that, in regard to responses to diabetes-related stigma, psychological and behavioural patterns of patients with T2DM could be explained by the following two dimensions: (1) a sense of self-esteem in relation to their illness and (2) attitudes towards social participation (for more details, see our previous paper $^{21}$ ). These psychological and behavioural patterns can be categorised as follows: group A (high self-esteem/high social participation), group B (high self-esteem/low social participation), group C (low self-esteem/high social participation) and group D (low self-esteem/low social participation). We qualitatively found that, compared with other groups, groups C and D seemed to develop lower self-esteem as a result of their own negative experiences and perceptions of their illness (stigma). The internalisation of this stigma (self-stigma) was seen as a possible contributing factor to changes in social participation and treatment adherence. ${ }^{21}$ However, the associations between these distinct psychological and behavioural patterns of stigma (self-esteem and social participation) with self-stigma, patient activation for engaging in self-care and glycaemic control remain unproven quantitatively. Therefore, we need to investigate further in order to detail the characteristics of the psychological and behavioural patterns of stigma in T2DM. Accordingly, the aim of this study was to test the psychological and behavioural patterns of stigma (self-esteem and social participation) and their relationship to self-stigma, patient activation for engaging in self-care and glycaemic control among patients with T2DM.

\section{METHODS}

\section{Study participants}

A questionnaire-based cross-sectional study was conducted between November 2013 and March 2014. Consecutive sampling was used to recruit all outpatients with T2DM who had visited an endocrinologist on a specific date at four locations in Japan (ie, two university hospitals, one non-university-affiliated hospital and one non-university-affiliated clinic). Patients were recruited through their physicians. After the physicians obtained permission, the patients received an explanation of the study's purpose by the research staff, after which written informed consent was obtained. Inclusion criteria were as follows: presence of T2DM, age $20-74$ years, ability to read and speak Japanese, no diagnosis of dementia and/or psychosis, and no need for urgent medical procedures or examinations. Patients completed a questionnaire that took $\sim 15-20 \mathrm{~min}$.

This study was approved in advance by the Research Ethics Committee of the University of Tokyo Graduate School of Medicine and Faculty of Medicine, and by each participating facility.

\section{Study measures}

Participants' sociodemographic factors included age, sex, education (in years) and marital status. We also collected participants' clinical information such as body mass index (BMI), time (in months) since diagnosis of T2DM, injection therapy, diabetes-related complications and glycated haemoglobin (HbAlc) level. We calculated the number of diabetes-related complications as the simple sum of six complications in reference to the Diabetes Complications Index. ${ }^{22}$ Scores ranged from 0 to 6 .

\section{Self-esteem}

We used the Rosenberg Self-Esteem Scale (SES) to assess self-esteem levels. ${ }^{18} 23$ The SES is a widely accepted scale due to its high reliability and validity. It contains 10 items scored on a four-point Likert scale, from 1 (strongly disagree) to 4 (strongly agree). Five negative items were reverse-scored to compute the total scores of individual participants. In this study, internal consistency was 0.79. All participants were assigned to one of two groups (high self-esteem/low self-esteem) depending on whether their score was above or below the total mean score. Since the self-esteem levels were normally distributed, the mean value of the score was used as the threshold.

\section{Social participation}

We assessed patients' levels of generic functional states using the following three subscales in the 36-question Short Form Health Survey (SF-36), Role Physical (RP), 
Role Emotional (RE) and Social Function $(\mathrm{SF}),{ }^{24}$ as a suitable approximation for attitudes towards social participation. In this study, we use the term social participation as it was defined in our previous study; essentially, social participation is defined as the degree to which patients are willing to participate in social life, which is influenced more by public stigma than by physical complications stemming from the severity of their condition. ${ }^{21}$ Therefore, we intentionally chose these subscales not for the purpose of detecting small changes that were clinically important, but for the purpose of assessing patients' functional states that were influenced by their illness-related negative experiences as well as their illnesses. The generic health status highly correlates with patients' limitations in terms of engaging in daily and social activities due to physical health, mental health or both, based on patients' objective and subjective ratings. ${ }^{24}$ Additionally, the SF-36 is a widely accepted valid health measure for estimating a specific disease burden, such as cardiac disease, asthma and diabetes.

The RP subscale contains four items on role limitations due to physical problems, and it evaluates the extent to which physical capabilities limit daily activities. The RE subscale contains three items on role limitations due to emotional problems, and it evaluates the extent and degree to which emotional problems interfere with work or other activities. The SF subscale contains two items on social functioning, and it evaluates the amount of time and extent to which emotional problems interfere with family, friends and other social interactions. For each subscale, item scores were coded according to the scoring algorithm, and then summed, transferred onto a scale from 0 to 100 and converted to norm-based scoring, with a mean set to $50 .{ }^{24}$ Thereafter, we simply added up the total of each subscale score. We calculated the total mean score by dividing the total score by the total number of participants. We tested each subscale score (RP, RE, SF) individually, and then tested the total score of the subscales to see if participants' distributions were the same. Since the participants' distributions remained unchanged whether the subscale scores were tested individually or totally, we decided, for the sake of expediency, to use the total of the subscale scores as the levels of social participation in this study. All participants were assigned to one of two groups (high social participation/low social participation) based on whether their score was above or below the total mean score. Since the total of these subscale scores was normally distributed, the mean value of the total score was used as the threshold.

\section{Psychological and behavioural patterns of stigma}

In our previous qualitative study, ${ }^{21}$ we found that psychological and behavioural patterns of stigma varied depending on levels of illness-related self-esteem and attitudes towards social participation in patients with T2DM. For quantitative consistency, in this study we used the SES to measure self-esteem levels and three SF-36 subscales to measure social participation. As described above, participants were classified into different psychological and behavioural patterns of stigma based on each scale score: participants with high self-esteem were assigned to either group A (those with high social participation) or group B (those with low social participation; figure 1), and participants with low self-esteem were assigned to either group C (those with high social participation) or group D (those with low social participation; figure 1).

\section{Outcome measures}

Self-stigma

The Self-Stigma Scale was used to assess patients' level of self-stigma. ${ }^{14}$ The reliability and validity of the scale's Japanese version (SSS-J) were reported previously. ${ }^{13}$ The 39-item SSS-J allows four responses on a Likert scale (strongly disagree, disagree, agree and strongly agree) scored $0,1,2$ and 3, respectively. Total scores are treated as continuous and range from 0 to 117 . Higher scores represent higher levels of self-stigma. In this study, the SSS-J had an internal consistency of 0.96 .

\section{Patient activation}

The Patient Activation Measure (PAM-13) was used to assess patients' activation levels for self-care. ${ }^{25-27}$ Patient activation is a concept that entails a comprehensive approach to several elements related to activation, including the patients' knowledge, skills, confidence and behaviours needed to manage their illness. Previous studies have indicated that the PAM-13 predicts a range of comprehensive behaviours, not just healthy behaviours (eg, exercise, diet) but also disease-specific selfmanagement behaviours (eg, keeping a written diary of glucose levels, taking diabetes medication as recommended by the physician). ${ }^{26} 28$

The PAM-13 is a clinically used, highly reliable and valid scale that consists of 13 questions on a Likert scale with five response categories scored 1 (strongly disagree), 2 (disagree), 3 (agree), 4 (strongly agree) or missing (not applicable), with a possible total score of 13-52. These scores were then converted to an interval scale $(0-100)$. A high score corresponds to a positive attitude towards necessary behavioural changes during the course of treatment. The Japanese version of the PAM-13 for mental health was used without including the words 'mental health' as stipulated by the scale's developer. ${ }^{26}$ In this study, the scale had an internal consistency of 0.85 .

\section{Glycated haemoglobin}

HbAlc was determined through blood samples taken that day.

\section{Statistical analyses}

Descriptive statistics were calculated using means and SDs or numbers and percentages based on the nature of the variables. To ensure the validity of the classification 
Figure 1 Psychological and behavioural patterns of stigma in patients with type 2 diabetes-a hypothesis generated from results of a qualitative study.

SF-36, 36-question Short Form Health Survey.

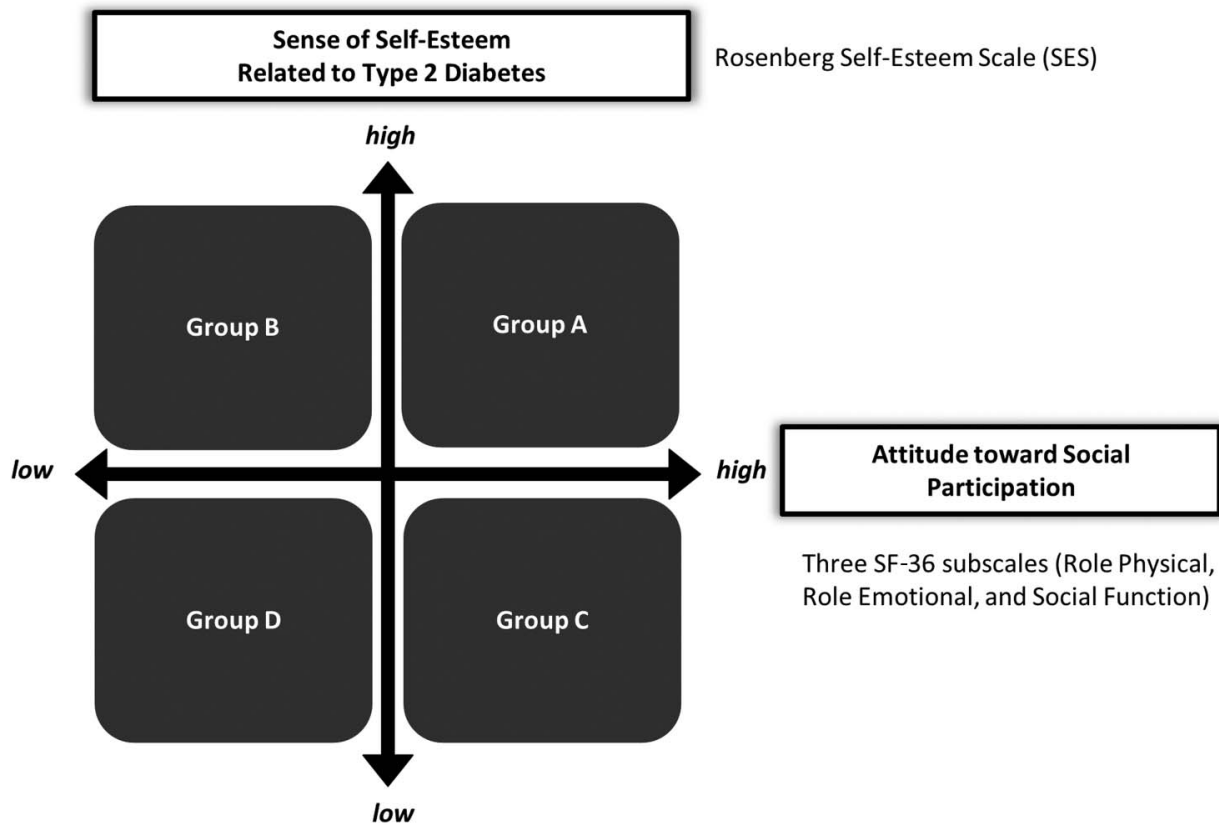

rationale of the four psychological and behavioural patterns of stigma in patients with T2DM, we calculated self-stigma levels using the SSS-J and then tested the respective self-stigma level within each of the four groups using a one-way analysis of covariance (ANCOVA) after controlling for age and sex. We then calculated means and 95\% CIs of PAM-13 scores and HbA1c levels for each group. We conducted a one-way ANCOVA to test for differences in mean scores of PAM-13 as well as those of HbA1c levels among the four groups, controlling for age and sex. Bonferroni correction was used to correct for multiple comparisons. All analyses were performed using SPSS V.23.0 (SPSS Japan, Tokyo, Japan).

\section{RESULTS}

We recruited 259 patients with T2DM through physicians, and obtained written informed consent from 218 patients (response rate $84.2 \%$ ). Among these participants, 217 completed the questionnaire (1 patient declined). In the analysis, we excluded five participants who chose 'strongly disagree' for all 39 items of the SSS-J based on their strong responses and uncertainty over whether the scale could measure what it was originally intended to assess. We also excluded three participants who chose 'strongly agree' for all 13 items of the PAM-13 as advised by the scale's developer. Therefore, 209 participants were included in our final analysis. The percentage of missing data was 0 for all questionnaire items.

Table 1 shows the sociodemographic and clinical characteristics of 209 participants. There were 168 male participants $(80.4 \%)$ and 41 female participants $(19.6 \%)$, with a mean age of $60.2 \pm 10.1$ years. Mean duration of T2DM was $159.1 \pm 113.8$ months, mean BMI was
Table 1 Sociodemographic and clinical characteristics of participants $(n=209)$

\begin{tabular}{lc}
\hline Patient characteristics & N (\%) or \\
mean $( \pm S D)$ \\
\hline Sex & \\
$\quad$ Male & $168(80.4)$ \\
$\quad$ Female & $41(19.6)$ \\
Age (years) & $60.2( \pm 10.1)$ \\
BMl (kg/m ${ }^{2}$ ) & $26.3( \pm 5.2)$ \\
Duration of diabetes (months) & $159.1( \pm 113.8)$ \\
Injection therapy & \\
$\quad$ Yes & $73(34.9)$ \\
$\quad$ No & $136(65.1)$ \\
Number of diabetes-related complications & $0.57( \pm 0.86)$ \\
(0-6) & \\
HbA1c (\%) & $7.3( \pm 1.2)$ \\
HbA1c (mmol/mol) & $56( \pm 13.1)$ \\
Education (years) & $13.9( \pm 2.3)$ \\
Marital status & \\
$\quad$ Married & $151(72.2)$ \\
$\quad$ Unmarried/divorced/bereaved & $58(27.8)$ \\
\hline *The Diabetes Complications Index. & \\
BMl, body mass index; HbA1c, glycated haemoglobin.
\end{tabular}

$26.3 \pm 5.2 \mathrm{~kg} / \mathrm{m}^{2}$ and mean HbA1c was $7.3 \pm 1.2 \% \quad(56$ $\pm 13.1 \mathrm{mmol} / \mathrm{mol})$. Mean number of diabetes-related complications was $0.57 \pm 0.86$, and $34.9 \%$ of participants received injection therapy (insulin or other injectable medications). Mean number of years of education was

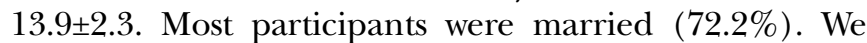
assessed the equivalence of sociodemographic and clinical variables (eg, education, marital status, BMI, duration of T2DM, diabetes-related complications) between the four groups; however, we did not find any differences between the four groups. 
Figure 2 shows mean self-stigma levels of different psychological and behavioural patterns of stigma. Using ANCOVA after controlling for age and sex, there was a significant difference in self-stigma levels between the four groups $(F[3,203]=15.70, p<0.001)$. We observed the highest mean self-stigma levels in group $\mathrm{D}$ followed in decreasing order by groups $\mathrm{C}, \mathrm{B}$ and $\mathrm{A}$. The lowest mean self-stigma levels was group A. Multiple comparisons using the Bonferroni test showed significant differences in self-stigma levels between between groups $\mathrm{C}$ and $\mathrm{D}(\mathrm{p}=0.01)$. The test also showed that there were no significant differences in self-stigma levels between groups $B$ and $C(p=1.0)$.
Figure 3 shows mean PAM-13 scores and HbA1c levels of different psychological and behavioural patterns of stigma. Using ANCOVA after controlling for age and sex, there was a significant difference in mean PAM-13 scores between the four groups $(\mathrm{F}[3,203]=9.34, \mathrm{p}<0.001)$. Multiple comparisons using the Bonferroni test showed significantly lower PAM-13 scores in group D compared with groups $A(p<0.001)$ and $B(p=0.02)$. As for HbA1c levels, using ANCOVA after controlling for age and sex, differences in means between the four groups showed a moderate increase from group $\mathrm{A}$ to group $\mathrm{D}$, using ANCOVA $(\mathrm{F}[3,203]=2.36, \mathrm{p}=0.07)$; however, there was no significant difference between groups $\mathrm{A}$ and $\mathrm{D}(\mathrm{p}=0.09)$.
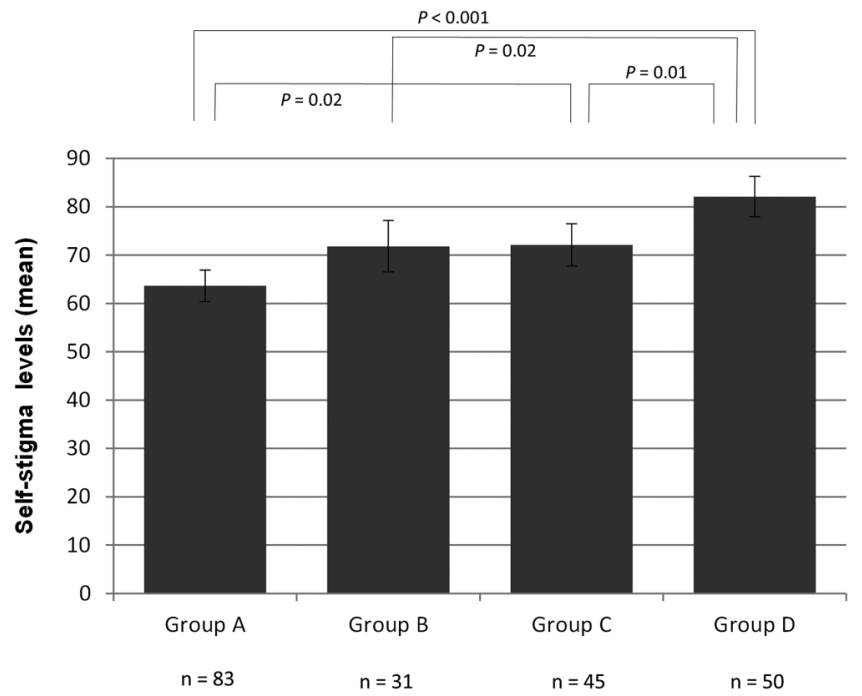

Figure 2 Mean self-stigma levels (SSS-J) of different psychological and behavioural patterns of stigma ( $n=209)$. Group A, high SES/high SF-36; Group B, high SES/low SF-36; Group C, low SES/high SF-36; Group D, low SES/low SF-36.

ANCOVA, analysis of covariance; SSS-J, Japanese version of Self-Stigma Scale; SES, Rosenberg Self-Esteem Scale; SF-36, 36-question Short Form Health Survey.
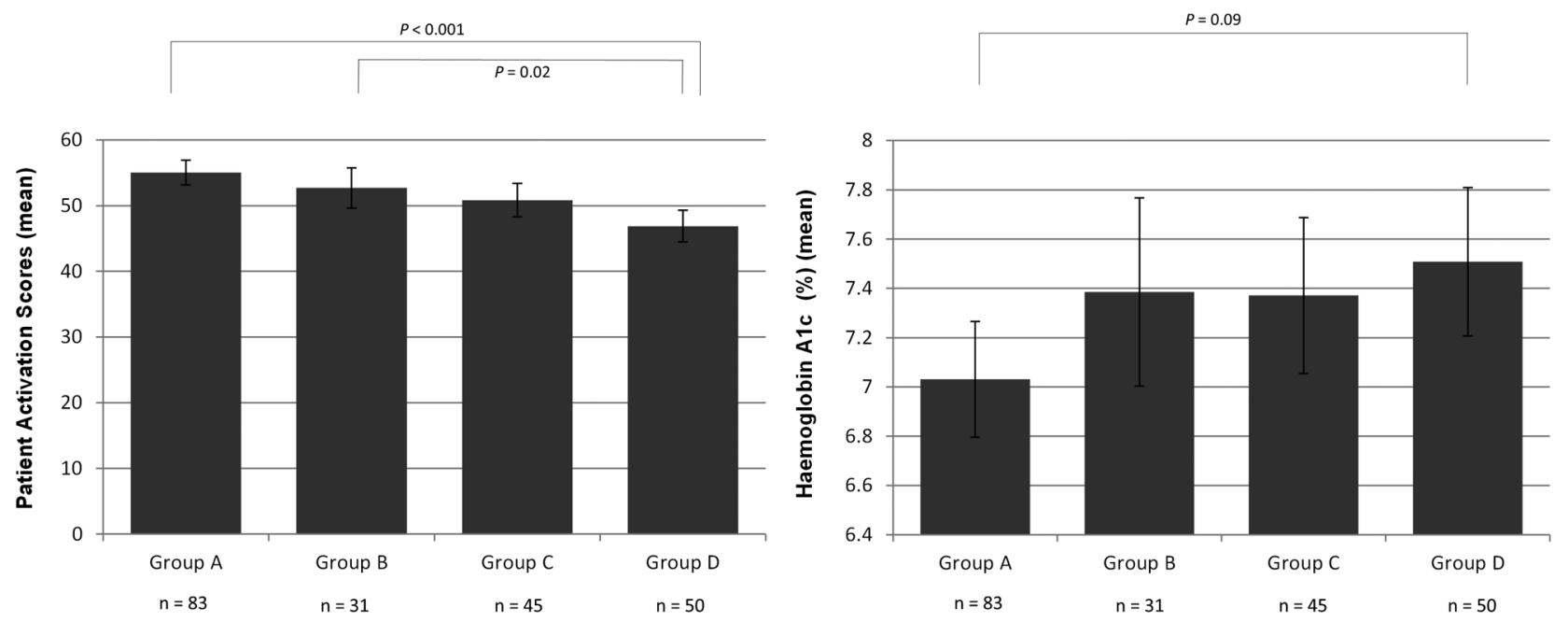

Figure 3 Mean patient activation scores (PAM-13) and HbA1c levels of different psychological and behavioural patterns of stigma ( $n=209$ ). Group A, high SES/high SF-36; Group B, high SES/low SF-36; Group C, low SES/high SF-36; Group D, low SES/low SF-36.

ANCOVA, analysis of covariance; PAM-13, Patient Activation Measure; HbA1c, glycated haemoglobin; SES, Rosenberg SelfEsteem Scale; SF-36, 36-question Short Form Health Survey. 


\section{DISCUSSION}

In this study, we aimed to test the psychological and behavioural patterns of stigma (self-esteem and social participation) and their relationship to self-stigma, patient activation and glycaemic control. The highest self-stigma levels were observed in group D, the group that exhibited low self-esteem and low social participation. The results concerning the association between low self-esteem and self-stigma are consistent with established theoretical perspectives on self-stigma. ${ }^{13} 19{ }^{20} \mathrm{On}$ the other hand, the results concerning the association between low social participation and self-stigma could be a novel finding regarding the characteristics of selfstigma among patients with T2DM. Thus, those who would fall in group D and exhibit that particular psychological and behavioural pattern may experience selfstigma and poorer patient activation for self-care.

The strengths of this study include its ability to delineate the psychological and behavioural patterns of self-stigma by the two dimensions (self-esteem and social participation) derived from our previous qualitative study, and then use objective measures through the Rosenberg SES and the SF-36 subscales to classify patients with T2DM into these groups. This study suggests that self-stigma includes both psychological states (self-esteem) and behavioural manifestations (social participation) among patients with T2DM. Without our previous analyses using qualitative data, we would not have been able to find a new dimension - the behavioural dimension-of self-stigma, or elucidate both the psychological manifestations (selfesteem) and behavioural manifestations (levels of social participation) of self-stigma in patients with T2DM.

This study has several limitations. First, since this study is cross-sectional, it can only demonstrate what the psychological and behavioural patterns of self-stigma look like, and their relationship to patient activation for selfcare; however, we do not know any causal relationships concerning each variable. For the next step, future research will be needed to investigate the causality of these variables to conceptualise self-stigma in patients with T2DM. Second, the amount of men in our sample outweighed the amount of women $(80.4 \%$ of the participants were male). Although the prevalence of T2DM is higher among men $(24.0 \%)$ than women $(13.4 \%)$ in Japan, ${ }^{29}$ it is not known exactly why we had such a large number of male participants compared with female participants. Third, this study did not include patients who were examined by primary care physicians in the community. Therefore, these findings do not capture the entire picture of patients with varying degrees of severity of T2DM. To verify the effects of self-stigma on the attitudes of patients with T2DM towards self-care management, further studies with a more representative population will be needed. Fourth, in this study, we used only three SF-36 subscales (RP, RE and SF) as suitable approximations to assess the level of social participation both physically and emotionally, although the subscales may not directly assess social participation itself. A new questionnaire needs to be developed with reliability and validity to assess social participation more accurately.

Our findings can have some important implications. Self-stigma can be observed by healthcare professionals during their daily practice. To help optimise the effectiveness of diabetes treatment, healthcare professionals could regularly assess whether patients are experiencing self-stigma and then help them develop positive personal images of their illness to enhance self-esteem. Additionally, for any patients who seem to limit their social participation regardless of the severity of the condition, healthcare professionals could closely examine and actively listen to patients' accounts of recent social activities and the reasoning behind their low social participation, and encourage them to find opportunities for social interaction as an important part of their treatment.

\section{Author affiliations}

${ }^{1}$ Department of Health and Social Behavior, School of Public Health, The University of Tokyo, Tokyo, Japan

${ }^{2}$ The Health Care Science Institute, Tokyo, Japan

${ }^{3}$ Department of Internal Medicine, School of Medicine, Teikyo University,

Tokyo, Japan

${ }^{4}$ Mitsui Memorial Hospital, Tokyo, Japan

${ }^{5}$ The Institute for Adult Diseases Asahi Life Foundation, Tokyo, Japan

${ }^{6}$ Department of Diabetes and Metabolic Diseases, Graduate School of

Medicine, The University of Tokyo, Tokyo, Japan

Correction notice This article has been corrected since it was first published. The author proof corrections were missed out of the original version and have now been added in. Commas have been added into the $F$ values throughout the paper to make them statistically correct.

Acknowledgements The authors would like to acknowledge all the physicians who helped recruit patients for this study. They are also grateful to the many graduate students who helped collect the data. Similarly, the authors would like to express their appreciation to all of the study participants.

Contributors AK conceptualised and designed the study. AK coordinated the study; acquired, analysed and interpreted the data; and prepared the paper. $\mathrm{HH}$ helped to analyse and interpret the data. $\mathrm{AK}$ and $\mathrm{HH}$ held primary responsibility for data access. YF, SF, AI, YO, RS, TY, KU and TK made significant contributions to the critical interpretation of results with regard to important practical content. All authors read and approved the final version of the manuscript.

Funding This research received no specific grant from any funding agency in the public, commercial or not-for-profit sectors.

Competing interests None declared

Patient consent Obtained.

Ethics approval The Research Ethics Committee of the University of Tokyo Graduate School of Medicine and Faculty of Medicine, and each participating facility.

Provenance and peer review Not commissioned; externally peer reviewed.

Data sharing statement No additional data are available.

Open Access This is an Open Access article distributed in accordance with the Creative Commons Attribution Non Commercial (CC BY-NC 4.0) license, which permits others to distribute, remix, adapt, build upon this work noncommercially, and license their derivative works on different terms, provided the original work is properly cited and the use is non-commercial. See: http:// creativecommons.org/licenses/by-nc/4.0/

\section{REFERENCES}

1. Schabert J, Browne JL, Mosely K, et al. Social stigma in diabetes: a framework to understand a growing problem for an increasing epidemic. Patient 2013;6:1-10. 
2. Tak-Ying Shiu A, Kwan JJ, Wong RY. Social stigma as a barrier to diabetes self-management: implications for multi-level interventions. $J$ Clin Nurs 2003;12:149-50.

3. Scollan-Koliopoulos M, O'Connell KA, Walker EA. Legacy of diabetes and self-care behavior. Res Nurs Health 2007;30:508-17.

4. Weiler DM, Crist JD. Diabetes self-management in a Latino social environment. Diabetes Educ 2009;35:285-92.

5. Singh $\mathrm{H}$, Cinnirella $\mathrm{M}$, Bradley $\mathrm{C}$. Support systems for and barriers to diabetes management in South Asians and Whites in the UK: qualitative study of patients' perspectives. BMJ Open 2012;14:e001459.

6. Browne JL, Ventura A, Mosely K, et al. 'I call it the blame and shame disease': a qualitative study about perceptions of social stigma surrounding type 2 diabetes. BMJ Open 2013;3:e003384.

7. Winkley K, Evwierhoma C, Amiel SA, et al. Patient explanations for non-attendance at structured diabetes education sessions for newly diagnosed type 2 diabetes: a qualitative study. Diabet Med 2015;321:120-8.

8. Hallgren EA, McElfish PA, Rubon-Chutaro J. Barriers and opportunities: a community-based participatory research study of health beliefs related to diabetes in a US Marshallese community. Diabetes Educ 2015;41:86-94.

9. Lee SM, Lim LC, Koh D. Stigma among workers attending a hospital specialist diabetes clinic. Occup Med (Lond) 2015;65:67-71.

10. Della LJ, Ashlock MZ, Basta TB. Social constructions of stigmatizing discourse around type 2 diabetes diagnoses in Appalachian Kentucky. Health Commun 2016;31:806-14.

11. Corrigan PW, Watson AC. The paradox of self-stigma and mental illness. Clin Psychol Sci Pr 2002;9:35-53.

12. Rüsch N, Angermeyer MC, Corrigan PW. Mental illness stigma: concepts, consequences, and initiative to reduce stigma. Eur Psychiatr 2005;20:529-39.

13. Kato $A$, Takada $M$, Hashimoto $H$. Reliability and validity of the Japanese version of the Self-Stigma Scale in patients with type 2 diabetes. Health Qual Life Outcomes 2014;12:179.

14. Mak WWS, Cheung RYM. Self-stigma among concealable minorities in Hong Kong: conceptualization and unified measurement. Am J Orthopsychiatry 2010;80:267-81.

15. Kato A, Fujimaki $Y$, Fujimori S, et al. Association between self-stigma and self-care behaviors in patients with type 2 diabetes: a cross-sectional study. BMJ Open Diabetes Res Care 2016;4: e000156.

16. Herzlich A. Health and illness: a social psychological analysis. London: Academic Press, 1973.
17. Radley A. Making sense of illness: the social psychology of health and disease. Thousand Oaks: Sage, 1994.

18. Rosenberg M. Society and adolescent self-image. Princeton: Princeton University Press, 1965.

19. Link BG, Struening EL, Neese-Todd S, et al. Stigma as a barrier to recovery: the consequence of stigma for the self-esteem of people with mental illness. Psychiatr Serv 2001;52:1621-6.

20. Corrigan PW, Watson AC, Barr L. The self-stigma of mental illness: implications for self-esteem and self-efficacy. J Soc Clin Psychol 2006;25:875-84.

21. Kato A, Fujimaki Y, Fujimori S, et al. A qualitative study on the impact of internalized stigma on type 2 diabetes self-management. Patient Educ Couns 2016:99:1233-9.

22. Fincke BG, Clark JA, Linzer M, et al. Assessment of long-term complications due to type 2 diabetes using patient self-report: the diabetes complications index. J Ambul Care Manage 2005;28:262-73.

23. Mimura C, Griffiths P. Japanese version of the Rosenberg Self-esteem Scale: translation and equivalence assessment. J Psychosom Res 2007;62:589-94.

24. Fukuhara S, Suzukamo Y. Health-related quality of life-SF8 and SF36. J Clin Exp Med 2005;213:133-6. (SF-36v2 ${ }^{\mathrm{TM}}$ Health SurveyC 1992, 2000, 2003 Quality Metric Incorporated, Medical Outcomes Trust and Shunichi Fukuhara. All rights reserved. SF-36® is a registered trademark of Medical Outcomes Trust. (SF-36v2 Standard, Japanese)

25. Fujita E, Kuno E, Kato D, et al. Development and validation of the Japanese version of the Patient Activation Measure 13 for Mental Health. Seishin Igaku (Clin Psychiatr) 2010;52:765-72.

26. Hibbard JH, Mahoney ER, Stockard J, et al. Development and testing of a short form of the Patient Activation Measure (PAM) Health Serv Res 2005;40:1918-30.

27. Hibbard JH, Stockard J, Mahoney ER, et al. Development of the Patient Activation Measure (PAM): conceptualizing and measuring activation in patient and consumers. Health Serv Res 2004;39:1005-26.

28. Hibbard JH, Mahoney ER, Stock R, et al. Do increases in patient activation result in improved self-management behaviors? Health Serv Res 2007;42:1443-63.

29. Mukai N, Doi Y, Ninomiya T, et al. Trends in the prevalence of type 2 diabetes and prediabetes in community-dwelling Japanese subjects: the Hisayama Study. J Diabetes Investig 2014;23:162-9. 